\title{
The critical current density of polycrystalline MgB2 prepared by using boron mixture
}

\begin{abstract}
In this study, boron powders with varying purity and form were mixed in different proportion to serve as precursors for reaction with $\mathrm{Mg}$ forming polycrystalline $\mathrm{MgB} 2$ bulks. The inductively measured superconducting transition temperature, Tc and the critical current density, $\mathbf{J}$ c were compared to that of samples prepared from the respective single boron. Overall, Tc remains largely unchanged for all samples. It was found that Jc at $6 \mathrm{~K}$ and $20 \mathrm{~K}$ did not degrade significantly up to $4.6 \mathrm{~T}$ as a result of adding impure boron as much as 10 wt.\% indicating comparable Jc can be obtained without dependence of use of expensive high purity boron powder alone. The systematic decrease of Jc with increasing impure boron additions shows that a compromise between desired and cost reduction can be made by varying the boron powder proportion. Finally, samples prepared from the mixture of both impure crystalline and amorphous borons even show enhanced Jc up to $3 \mathrm{~T}$ at $20 \mathrm{~K}$. The increase in Jc correlates with the retention of strain level in these samples probably resulted from the more similar reaction rate of the respective borons.
\end{abstract}

Keyword: Boron precursors, Critical current density, MgB2, Strain 